\title{
Theoretical Investigation of Geometric Configurations and Vibrational Spectra in Citric Acid Complexes
}

\author{
Rodrigo Marques Ferreira ${ }^{\mathrm{a}}$, Maycon Motta ${ }^{\mathrm{a}, \mathrm{b}}$, Augusto Batagin-Neto ${ }^{\mathrm{a}}$, \\ Carlos Frederico de Oliveira Graeff ${ }^{c}$, Paulo Noronha Lisboa-Filho ${ }^{c}$, Francisco Carlos Lavarda ${ }^{\text {** }}$

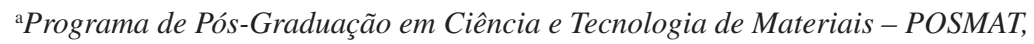 \\ Universidade Estadual Paulista - UNESP, Bauru, SP, Brazil \\ ${ }^{\mathrm{b}}$ Grupo de Supercondutividade e Magnetismo, Departamento de Física, \\ Universidade Federal de São Carlos - UFSCar, São Carlos, SP, Brazil \\ ${ }^{ }$Departamento de Física, Faculdade de Ciências, \\ Universidade Estadual Paulista - UNESP, Bauru, SP, Brazil
}

Received: April 15, 2013; Revised: March 22, 2014

\begin{abstract}
The performance of advanced electronic ceramics is directly related to the synthesis route employed. Sol-gel methods are widely used for this purpose. However, the physicochemical intermediate steps are still not well understood. Better understanding and control of these processes can improve the final quality of samples. In this work, we studied theoretically the formation of metal complexes between citric acid and lithium or barium metal cations with different citric acid/metal proportions, using Density Functional Theory electronic structure calculations. Infrared and Raman scattering spectra were simulated for the more stable geometric configurations. Using this methodology, we identified some features of complexes formed in the synthesis process. Our results show that the complexes can be distinguished by changes in the bands assigned to $\mathrm{C}=\mathrm{O}, \mathrm{COH}-$, and $\mathrm{COO}^{-}$group vibrations. An estimate of the most stable complexes is made based on total energy.
\end{abstract}

Keywords: advanced electronic ceramics, density functional theory, infrared spectra simulations, Raman spectra simulations, sol-gel, barium complex

\section{Introduction}

The performance of advanced electronic ceramics is highly dependent on their synthesis route. In the so-called sol-gel process, there are many variations in the routes used for preparing samples. A deeper understanding of complexes between $\alpha$-hydroxycarboxylic acids and metals $(\mathrm{M})$ that are formed on those routes is desirable, since these compounds are highly effective in obtaining heterometallic advanced ceramics ${ }^{1}$. Several metallic ions with different applications have been employed in this kind of route, such as lithium ion in the manufacturing of cathodes for fuel cells ${ }^{2,3}$ and barium ion in pellets of high temperature superconductors ${ }^{4-6}$, magnetic materials $\mathrm{s}^{7,8}$, and multiferroic materials ${ }^{9}$.

The modified polymeric precursors method (MPPM) ${ }^{1,4}$ involves the polyesterification process of a metal chelate complex using a hydroxycarboxylic acid and a polyhydroxy alcohol in an aqueous solution, which turns into a polymeric gel. In this method, the most commonly employed carboxylic acid is citric acid (CA) ${ }^{4}$. The MPPM method has been widely used, since it allows for the preparation of polycrystalline samples of excellent quality, obtaining a highly homogeneous ceramic powder with nanometer-scale particles, and it is reactive at temperatures up to $30 \%$ lower than the ceramic powders obtained by traditional routes ${ }^{1,5}$.

*e-mail: lavarda@fc.unesp.br
Several steps are involved in the MPPM procedure. As mentioned, the main goal of this method is to obtain a ceramic powder using polymeric precursors via $\alpha$-hydroxycarboxylic acid and ethylene glycol. There are two sequential chemical reactions involved in these methods ${ }^{1}$. The first, which is the purpose of our theoretical studies, is the chelation between the $\alpha$-hydroxycarboxylic acid and metal ions obtained from simple salts, such as oxides or carbonates. The second is the polyesterification reaction with ethylene glycol, which results in a polymer. These reactions occur in an aqueous medium to form a sol. The gel is obtained after the evaporation of water by raising the solution temperature to $90^{\circ} \mathrm{C}$. Following this, heat treatment at $400^{\circ} \mathrm{C}$ is carried out on the polymer, resulting in a multicomponent oxide ceramic powder with extremely homogeneous stoichiometry. The resulted powder is then calcinated with several intermediate grindings in order to remove the residual organic matter and form the desired final phase. After calcination, the powder is pressed and sintered to obtain ceramic pellets.

The polymer formed during synthesis is responsible for both the intermediate and final structures, since it is believed that the complexes tend not to break down during the polymerization process. Consequently, better understanding of the formation of metal complexes could provide valuable information for improving the quality of the final samples. 
Although it is a widely used synthesis route, intermediate physicochemical reactions and metal complexes are still poorly known ${ }^{6}$, having been the subject of only a small number of theoretical works ${ }^{10-12}$.

In this report, we have studied theoretically the citric acid complexes formed by the lithium and barium ions. For the lithium complexes, one citric acid molecule was chelated with one or two metallic ions, whereas one barium ion was chelated with one, two, or three citric acid molecules in the barium complexes. The identification of geometry configurations, the associated bands for infrared (IR) and Raman spectra and the stability of such metallic ion complexes were investigated, providing additional information that can aid the preparation of electronic ceramics.

\section{Methodology}

Our work has two distinct steps for each system studied. The first step is determining the most stable structure (geometry optimization) and the second is simulating infrared and Raman shift spectra.

Prior to setting up initial geometries for further optimization, Condensed-to-Atoms Fukui Indices analysis (CAFIs $)^{13}$ was carried out to evaluate the most likely chelation sites for both metal ions on the CA structure. In general, these indices describe how the frontier orbitals are modified when the number of electrons is changed and are employed to understand and predict the reactivity of molecules and polymers ${ }^{14-16}$. According to the type of reaction, three distinct CAFIs are defined. In our case (metal incorporation), we considered electrophilic attack $\left(\mathrm{f}_{\mathrm{k}}^{-}\right)$analysis:

$\mathrm{f}_{\mathrm{k}}^{-}=\mathrm{q}_{\mathrm{k}}(\mathrm{n})-\mathrm{q}_{\mathrm{k}}(\mathrm{N}-1)$

for atom $\mathrm{k}$ as a nucleophile, where $\mathrm{q}_{\mathrm{k}}(\mathrm{n})$ and $\mathrm{q}_{\mathrm{k}}(\mathrm{N}-1)$ are the electronic populations on the $\mathrm{k}$-th atom of the cationic and neutral species of the system studied, calculated for neutral specie geometry.

After CAFIs analysis, the initial geometries of the metal complexes were then optimized using the semiempirical Parametric Method $6{ }^{17}$ (PM6), implemented in package MOPAC $2009^{18}$. This method provides a very good structure to be the initial geometrical configuration from which to start the following phase, the ab initio quality search for the most stable structure, for which we employed the Density Functional Theory (DFT) ${ }^{19}$. For DFT calculations, the most appropriate functional must be selected to ensure highquality results. Of the many available functionals, we chose the hybrid functional B3LYP ${ }^{20}$, which has already been used successfully in similar compounds ${ }^{20,21}$. Moreover, this functional is widely used, since it has proven to be reliable, generally yielding good-quality results ${ }^{22}$. The $6-31 \mathrm{G}$ basis functions $\operatorname{set}^{23}$ was adopted for all atoms except barium, for which we adopted the set LANL2DZ-ECP ${ }^{24,25}$, owing to the high number of electrons of this metallic element. CAFIs, Raman, and IR spectra were calculated with the same DFT approach used in the geometry optimization.

In CAFIs calculations, the electronic populations were evaluated by electrostatic potential fitting $(\mathrm{ESP})^{26}$ and Mulliken population analysis ${ }^{27}$, and the restricted open-shell approach (ROKS) was employed to avoid spin contamination problems.

After obtaining the most stable structure for each complex, we simulated the infrared and Raman shift spectra to compare with the experimental data. The calculated frequency values are usually multiplied by a scaling factor, which varies according to the theoretical model used. For DFT/B3LYP/6-31G and DFT/B3LYP/LANL2DZ, the scale factors are 0.962 and 0.961 , respectively ${ }^{28}$. The results presented in this work were not multiplied by a scaling factor.

All DFT calculations were performed with restricted electronic orbitals, in vacuum, with the General Atomic and Molecular Electronic Structure System (GAMESS) package $^{29}$. The software employed for the visualization of complex structures and the evaluation of their properties was Gabedit ${ }^{30}$ and Wxmacmolplt ${ }^{31}$.

\section{Results and Discussion}

First, it was necessary to define an initial geometry for each complex studied. For this purpose, we needed to know the oxidation state of the metal ions and the deprotonation sites of citric acid. It was considered that lithium could enter as a monovalent cation $\left(\mathrm{Li}^{+1}\right)$ and barium as a divalent cation $\left(\mathrm{Ba}^{+2}\right)^{32}$. Deprotonation of CA was made, such that the final complex was a closed-shell system.

Figure 1 shows the structure of CA and the CAFIs calculated for eletrophilic attack employing Mulliken population analysis. Regions in red and blue represent reactive and non-reactive sites, respectively. Other colors indicate sites with intermediary reactivity. Similar results were obtained via ESP charge partitioning.

As can be seen, the central carboxylic group shows higher CAFIs for eletrophilic attacks, defining a susceptible region for metal chelation. Based on this result, the first proton $\left(\mathrm{H}^{+}\right)$was removed from this group in all calculations.

In the complexes of citric acid with lithium, one $\mathrm{H}^{+}$ion was removed from $\mathrm{CA}$ for each lithium ion present. In the case of $\mathrm{CA} / \mathrm{Li}$, after removing the first proton from $\mathrm{CA}$, we added one lithium ion $\mathrm{Li}^{+1}$ near the molecule. The complex $\mathrm{CA} / 2 \mathrm{Li}$ was modelled after the complex $\mathrm{CA} / \mathrm{Li}$ optimization by subtracting a second $\mathrm{H}^{+}$from one of the side carboxylic groups of the CA molecule. We then added a second lithium ion near the complex. The obtained stable final structures are shown in Figure 2 for both citric acid/metal ratios, and agree well with previous results ${ }^{33}$.
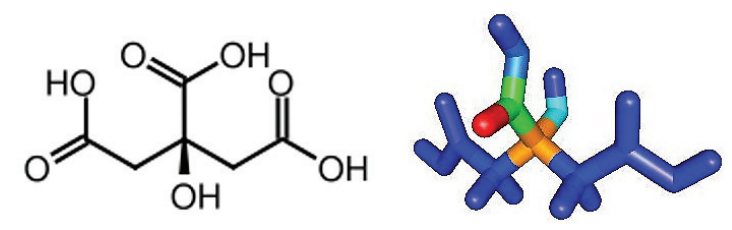

Figure 1. Citric acid structural formula and Condensed-to-Atoms Fukui Indices analysis. The most likely site for an electrophilic attack is shown in red (color online). 
$\mathrm{CA} / \mathrm{Li}$

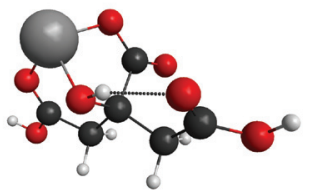

(a)

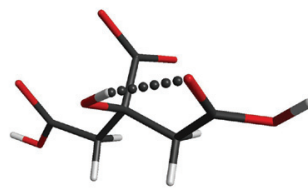

$\mathrm{CA} / 2 \mathrm{Li}$

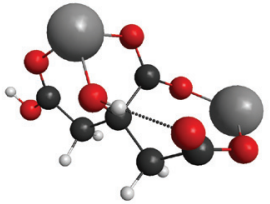

(b)
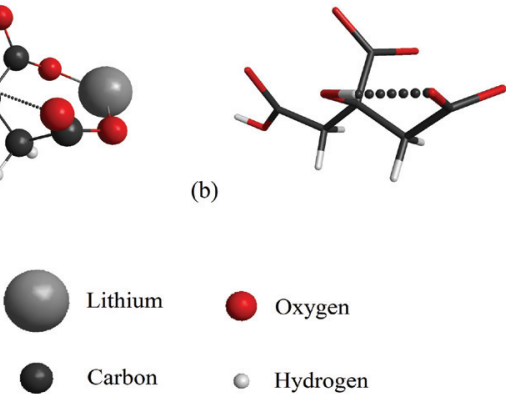

Hydrogen

Figure 2. Optimized structures of complexes formed by one molecule of citric acid with (a) one and (b) two lithium atoms; the citric acid model is also shown for each complex (color online).

The results obtained for the infrared spectra are shown in Figure 3. For a pure CA molecule, it is possible to observe bands corresponding to the vibrations of the groups $\mathrm{O}-\mathrm{H}$ (stretching) at $3650 \mathrm{~cm}^{-1}, \mathrm{CH} 2$ (stretching) at $3140 \mathrm{~cm}^{-1}, \mathrm{C}=\mathrm{O}$ (stretching) at $1750 \mathrm{~cm}^{-1}, \mathrm{COOH}$ (symmetrical stretching), $\mathrm{O}-\mathrm{H}$ (in-plane bending), and $\mathrm{C}-\mathrm{H}$ (wagging) between $1550 \mathrm{~cm}^{-1}$ and $1300 \mathrm{~cm}^{-1}, \mathrm{C}-\mathrm{OH}$ (stretching) at $1120 \mathrm{~cm}^{-1}, \mathrm{C}-\mathrm{OH}$ (wagging) at $685 \mathrm{~cm}^{-1}$ and $\mathrm{COOH}$ (scissoring) at $560 \mathrm{~cm}^{-1[34]}$. Our results show excellent agreement with experimental data ${ }^{35}$. For example, the $\mathrm{C}=\mathrm{O}, \mathrm{C}-\mathrm{OH}$ and $\mathrm{COOH}\left(1420 \mathrm{~cm}^{-1}\right)$ bands show a deviation of less than $1 \%$.

Figure 3 shows the influence of the number of lithium ions in the complex. One effect of the addition of Li occurs in bands associated with $\mathrm{C}=\mathrm{O}$ bonds. In pure $\mathrm{CA}$, this strong band is at $1750 \mathrm{~cm}^{-1}$, shifting to $1714 \mathrm{~cm}^{-1}$ and $1706 \mathrm{~cm}^{-1}$ when one or two lithium ions are added, respectively, owing to the deprotonation of the carboxyl groups. Furthermore, it is possible to see the presence of a new band at $1275 \mathrm{~cm}^{-1}$ for the $\mathrm{CA} / \mathrm{Li}$ complex, corresponding to the symmetric stretching of the $\mathrm{COO}^{-}$group. The band appears when carboxyl acts as a monodentate ligand ${ }^{36,37}$; this gives rise at the same time to an asymmetric stretching of the $\mathrm{COO}$ group at $1684 \mathrm{~cm}^{-1}$, which is hidden because of the strong vibrational mode of the $\mathrm{C}=\mathrm{O}$. On the other hand, a carboxyl group acts as a bidentate ligand in the $\mathrm{CA} / 2 \mathrm{Li}$ complex, with peaks at 1375 and $1631 \mathrm{~cm}^{-1[36-38]}$. The difference between the asymmetrical and symmetrical stretching of the $\mathrm{COO}$ group for the $\mathrm{CA} / \mathrm{Li}$ and $\mathrm{CA} / 2 \mathrm{Li}$ is in agreement with the criteria presented by Kakihana et al. ${ }^{37}$ for the bidentate or a monodentate character of the carboxylic group, compared with a free carboxylic group $\left(\mathrm{COO}^{-}\right)$, the difference between the asymmetric and symmetric stretching vibrations being $298 \mathrm{~cm}^{-1}\left(1702 \mathrm{~cm}^{-1}\right.$ and $1404 \mathrm{~cm}^{-1}$, respectively).

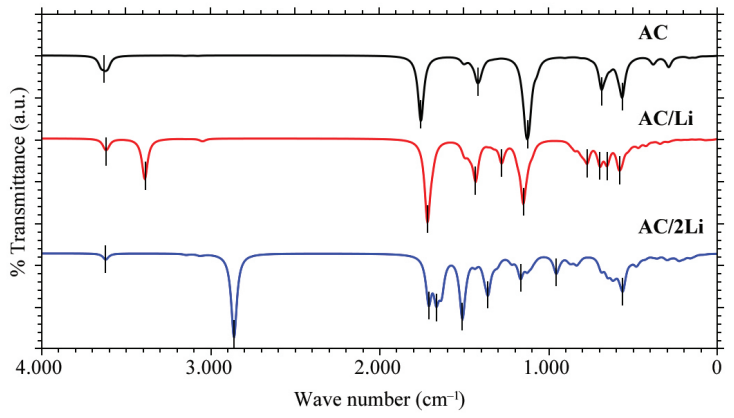

Figure 3. Simulation of infrared spectra for pure citric acid and the complexes with one and two lithium ions (color online).

Table 1. Bond orders for selected groups.

\begin{tabular}{ccc}
\hline $\begin{array}{c}\text { Bond } \\
\text { complex }\end{array}$ & $\mathbf{C}=\mathbf{O}$ & $\begin{array}{c}\text { OH } \\
\text { (hydrogen bond) }\end{array}$ \\
\hline $\mathrm{AC} / \mathrm{Li}$ & 1.735 & 0.113 \\
$\mathrm{AC} / 2 \mathrm{Li}$ & 1.506 & 0.202 \\
\hline
\end{tabular}

Another clear case of the influence of lithium appears in bands associated with $\mathrm{O}-\mathrm{H}$ and $\mathrm{COH}$ bonds, owing to a hydrogen bond that appears between the hydrogen of the hydroxyl $(-\mathrm{OH})$ group and the oxygen of the carbonyl $(\mathrm{C}=\mathrm{O})$, which belongs to a carboxylic group ${ }^{37}$, as shown in Figure 2 by a dotted line. In the spectrum of CA, the band associated with stretching vibrations of hydroxyl is located at $3630 \mathrm{~cm}^{-1}$. The introduction of one lithium ion causes a molecule distortion and a new band associated with the $\mathrm{OH}$ group appears at $3380 \mathrm{~cm}^{-1}$. This group is bonded to the $\mathrm{Li}$ ion ( $\mathrm{Li}-\mathrm{O}-\mathrm{H})$ and becomes weak bonded with the neighbor carboxylate through a hydrogen bond. This influence becomes stronger and shifts the band to $2860 \mathrm{~cm}^{-1}$ when two lithium ions are present. Moreover, there is an increase in the $\mathrm{CO}$ stretching vibration. In CA, the band associated with such bonds is located at $1129 \mathrm{~cm}^{-1}$ and the maximum shift is $1147 \mathrm{~cm}^{-1}$ and $1163 \mathrm{~cm}^{-1}$ in CA/Li and CA/2Li complexes, respectively. This means that there is competition between the hydrogen bond and the $\mathrm{COH}$ bond. The calculated bond order (which gives the strength of a bond) seems to corroborate this conclusion. In Table 1, we can see that, as the content of $\mathrm{Li}$ increases, the $\mathrm{C}=\mathrm{O}$ bond becomes weaker and the hydrogen bond becomes stronger.

The amount of lithium interferes with the bands in the region between 500 and $1000 \mathrm{~cm}^{-1}$. In CA, the bands in this region are well defined, with vibrations at $560 \mathrm{~cm}^{-1}$ and $685 \mathrm{~cm}^{-1}(\mathrm{COOH}$ and $\mathrm{COH})$, whereas new medium peaks can be observed at $630 \mathrm{~cm}^{-1}$ owing to the LiO stretching modes. As the number of lithium ions increases, there is an increase in the number of bands, which will then overlap.

Figure 4 shows the Raman bands for the CA molecule. The intense vibration at $3506 \mathrm{~cm}^{-1}$ is associated with the asymmetric stretching of $\mathrm{OH}$. It is also possible to observe the $\mathrm{CH} 2$ symmetric and asymmetric stretching at $3120 \mathrm{~cm}^{-1}$ and $3085 \mathrm{~cm}^{-1}$, respectively. Other minor bands are also observed near $1480 \mathrm{~cm}^{-1}$ for the $\mathrm{CH}_{2}$ symmetric stretching 


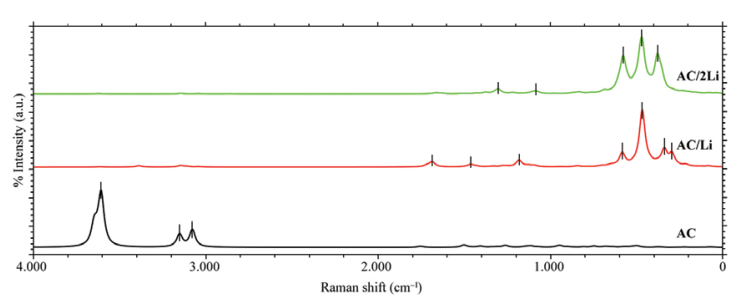

Figure 4. Simulation of Raman spectra for pure citric acid and complexes with one and two lithium ions (color online).

and further bands appear at $1444 \mathrm{~cm}^{-1}$ and $1175 \mathrm{~cm}^{-1}$ that are also associated with $\mathrm{CH}_{2}$. In the spectra of complexes of $\mathrm{CA} / \mathrm{Li}$ and $\mathrm{CA} / 2 \mathrm{Li}$, we observe $\mathrm{LiO}$ vibrations in the region between 300 and $600 \mathrm{~cm}^{-1}$ (see Figure 4).

Comparison of the simulated Raman spectra of lithium and CA complexes with pure CA shows that the intense bands located in the region between 3600 and $3000 \mathrm{~cm}^{-1}$ in pure CA practically disappear in the complexes. The interval 600-300 $\mathrm{cm}^{-1}$, which in pure CA shows some bands that are rather weak and not well defined, in the complexes has well defined and intense bands; as the amount of metal increases, these bands become more intense. The bands are attributed to the vibrations of the bonds between the lithium and oxygen atoms, as previously stated.

The marked differences between the complexes $\mathrm{CA} / \mathrm{Li}$ and $\mathrm{CA} / 2 \mathrm{Li}$ are better observed in the infrared spectra. The Raman spectra are very similar, revealing few distinctions except with regard to the intensities of the bands, which it is not possible to determine experimentally. Finally, from these observations we can say that the band that better distinguishes the complexes $\mathrm{CA} / \mathrm{Li}$ and $\mathrm{CA} / 2 \mathrm{Li}$ is located at $1275 \mathrm{~cm}^{-1}$ and $1355 \mathrm{~cm}^{-1}$, respectively, which refers to the ligand character of the carboxylate group. Moreover, the band at $2851 \mathrm{~cm}^{-1}$ in the infrared spectrum of the complex $\mathrm{CA} / 2 \mathrm{Li}$ indicates a stronger hydrogen bond of the $\mathrm{OH}$ stretching vibration. However, it is known that this region is experimentally masked because of the interference of $\mathrm{H}_{2} \mathrm{O}$ present in the air or absorbed on the surface of the sample while spectroscopy is performed.

In complexes between citric acid and barium, two $\mathrm{H}^{+}$ were subtracted from carboxyl groups for each barium ion present. Considering the metallic ion size, as expected, the CA molecule was less distorted with $\mathrm{Ba}^{2+}$ than it was with $\mathrm{Li}^{+}$ (1.35 and $0.68 \AA$ atomic radii, respectively), which allowed for the formation of hydrogen bonds to fully minimize the energy. Figure 5 presents the optimized structures of the different ratios between $\mathrm{CA} / \mathrm{Ba}$ complexes, which are $1 / 1$, $2 / 1$, and $3 / 1$. Kakihana and Yoshimura ${ }^{39}$ found that the CA/ Metal ratio 3/1 is the most stable.

Figure 6 shows a comparison of the simulated infrared spectra of pure $\mathrm{CA}, \mathrm{CA} / \mathrm{Ba}, 2 \mathrm{CA} / \mathrm{Ba}$, and $3 \mathrm{CA} /$ $\mathrm{Ba}$ complexes. Note that each proportion has its own characteristics. The region between $3500 \mathrm{~cm}^{-1}$ and $2250 \mathrm{~cm}^{-1}$ refers to the $\mathrm{OH}$ stretching vibrations. The band around $3600 \mathrm{~cm}^{-1}$ is due to free $\mathrm{OH}$ bonds, whereas the peaks at $3500 \mathrm{~cm}^{-1}, 3000 \mathrm{~cm}^{-1}$, and $2500 \mathrm{~cm}^{-1}$ for the $2 \mathrm{CA} / \mathrm{Ba}$ complex and at $3500-3250 \mathrm{~cm}^{-1}$ and $2245 \mathrm{~cm}^{-1}$ refer to $\mathrm{OH}$ influenced by hydrogen bonds. Experimentally, these
$\mathrm{CA} / \mathrm{Ba}$

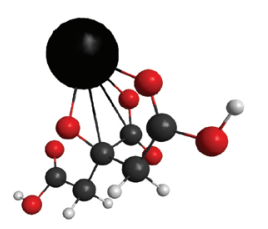

(a)

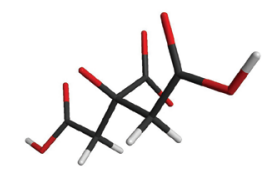

$2 \mathrm{CA} / \mathrm{Ba}$
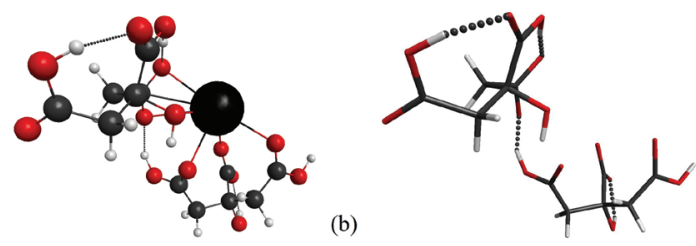

$3 \mathrm{CA} / \mathrm{Ba}$

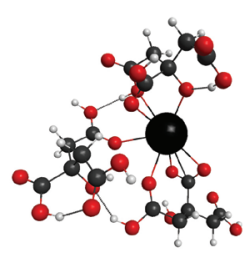

(c)

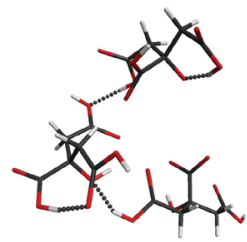

Oxygen

- Hydrogen

Figure 5. Optimized structure for the complex of a barium atom with (a) one, (b) two, and (c) three molecules of citric acid; the citric acid model is also shown for each complex (color online).

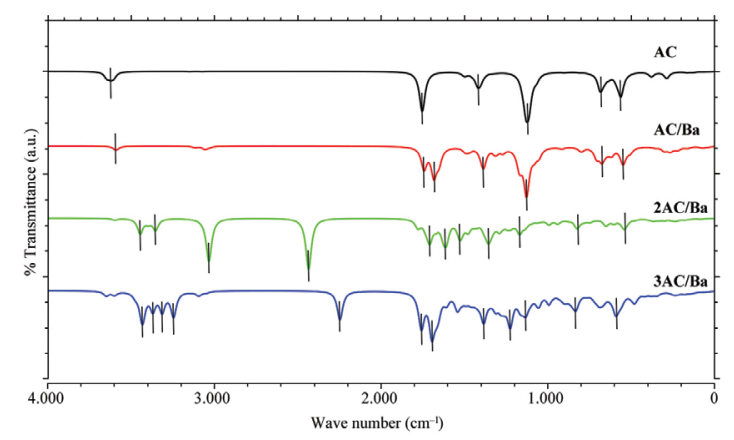

Figure 6. Simulation of IR absorption spectra for pure citric acid and complexes of barium (color online).

bands are hidden because of the water vibrations and adsorbed $\mathrm{CO}_{2}{ }^{[34]}$. Furthermore, the spectrum for the $\mathrm{CA} /$ $\mathrm{Ba}$ complex shows the same shift towards smaller values of wavenumbers for the $\mathrm{C}=\mathrm{O}$ bond at $1730 \mathrm{~cm}^{-1}$ as in the $\mathrm{CA} / \mathrm{Li}$ complex, and $2 \mathrm{AC} / \mathrm{Ba}$ and $3 \mathrm{AC} / \mathrm{Ba}$ show a defined peak at $1750 \mathrm{~cm}^{-1}$, associated with the presence of more $\mathrm{COOH}$ groups. The asymmetrical and symmetrical $\mathrm{COO}$ stretching shows a characteristic monodentate ligand for $\mathrm{CA} / \mathrm{Ba}\left(1683 \mathrm{~cm}^{-1}\right.$ and $\left.1269 \mathrm{~cm}^{-1}\right)$, monodentate for $2 \mathrm{AC} /$ 


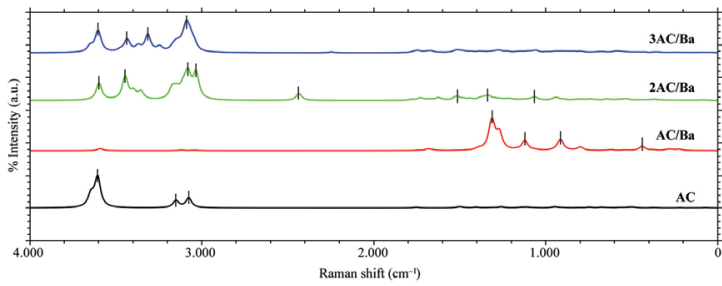

Figure 7. Simulation of Raman spectra for pure citric acid and complexes of barium (color online).

$\mathrm{Ba}\left(1707 \mathrm{~cm}^{-1}\right.$ and $\left.1290 \mathrm{~cm}^{-1}\right)$, and bidentate for $3 \mathrm{AC} / \mathrm{Ba}$ $\left(1606 \mathrm{~cm}^{-1} \text { and } 1383 \mathrm{~cm}^{-1}\right)^{37}$. Moreover, when the number of citric acid molecules increases, the quantity of $\mathrm{BaO}$ and hydrogen bonds also increases, which creates a larger distortion of the complex.

Another effect occurs in the bands associated with the $\mathrm{OH}, \mathrm{CH}$, and $\mathrm{COH}$ bonds spread in the interval from 1000 to $1500 \mathrm{~cm}^{-1}$. In pure CA, these bands are well defined and intense once all bonds are vibrating with nearly the same frequency, as described above. With the addition of $\mathrm{Ba}^{2+}$ ion, some bonds experience more interference than others, and this causes them to vibrate at different frequencies. This effect can be observed in the spectra of the $2 \mathrm{CA} / \mathrm{Ba}$ and $3 \mathrm{CA} /$ Ba complexes through the spreading and decreasing of band intensities related to these bonds. Further, vibrations below $1000 \mathrm{~cm}^{-1}$ due to $\mathrm{COOH}$ (scissoring), $\mathrm{COH}$ (wagging), and $\mathrm{BaO}$ are spread out in this region.

Figure 7 shows a comparison of the Raman spectra for the three simulated proportions. We can identify some unique features in each case. In the CA/Ba case, unlike the other cases, the spectrum shows the most intense bands in the region within the range of $750-1500 \mathrm{~cm}^{-1}$. These bands, as previously stated, are related to $\mathrm{COH}, \mathrm{CCO}$, and $\mathrm{BaO}$ vibrations. As we have concluded for the $\mathrm{CA}+\mathrm{Li}$ complexes, the identification of the different types of $\mathrm{CA}+\mathrm{Ba}$ complexes can be better accomplished by comparisons of IR spectra.

An estimate for the most stable lithium and barium citrates based on the total energy of the complexes was done using the following expressions:

$\Delta \mathrm{E}=(\mathrm{CA} / \mathrm{nLi})_{\text {total energy }}-\mathrm{n} \cdot\left(\mathrm{Li}^{+}\right)_{\text {total energy }}-\mathrm{K}_{1}$

and

$\Delta \mathrm{E}=(\mathrm{nCA} / \mathrm{Ba})_{\text {total energy }}-\mathrm{n} \cdot(\mathrm{CA})_{\text {total energy }}-\mathrm{K}_{2}$

where $\mathrm{n}$ is the number of $\mathrm{Li}^{+}$ions $(1,2)$ or is the number of CA molecules $(1,2,3)$ in the complexes. As $\mathrm{K}_{1}$ and $\mathrm{K}_{2}$ are constants, which are irrelevant to comparison of the different complexes, they were set to zero; $\mathrm{K}_{1}=(\mathrm{CA})_{\text {total energy }}+\mathrm{n} \cdot\left(\mathrm{H}^{+}\right)_{\text {total energy }}$ and $\mathrm{K}_{2}=2 \cdot\left(\mathrm{H}^{+}\right)_{\text {total energy }}+\left(\mathrm{Ba}^{+2}\right)_{\text {total energy }}\left(\right.$ where $\left(\mathrm{H}^{+}\right)_{\text {total energy }}$ is equal to zero). $\Delta \mathrm{E}$ is the variation of total energy between the complex and the complex's components: the larger $|\Delta \mathrm{E}|$ indicates the most stable complex. The results, shown in Figure 8 , indicate that $\mathrm{AC} / \mathrm{Li}$ and $3 \mathrm{CA} / \mathrm{Ba}$ are probably the most stable complexes, which in the case of barium citrates is in agreement with experimental findings ${ }^{39}$.

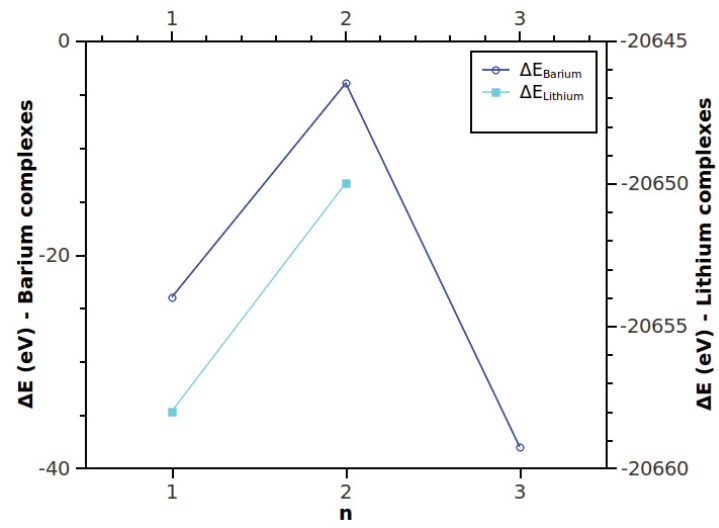

Figure 8. Total energy difference estimate for the most stable lithium and barium citrates complexes (color online).

Altogether, our results provide information about the intermediate complexes formed in the MPPM synthesis route. However, it is well known that in an experimental spectrum certain factors have an influence, which were not taken into account in our simulations, such as the interference of the solvent, the presence of impurities, and contaminants such as $\mathrm{H}_{2} \mathrm{O}$ and $\mathrm{CO}_{2}$. Thus, we believe that, although the bands we mention may be disguised by these interferences, they remain prominent in experimental results and help to distinguish the different complexes.

\section{Conclusions}

In this study, we presented a set of features for lithium and barium citric acid complexes that allow geometric configurations to be identified from comparisons with the associated bands for infrared and Raman spectra. In this context, IR is preferred over Raman spectra, as it offers features that enable better comparisons. The main features are associated with the vibrations of the groups $\mathrm{OH}, \mathrm{C}=\mathrm{O}$, $\mathrm{COOH}, \mathrm{COO}^{-}, \mathrm{COH}$ and $\mathrm{M}-\mathrm{O}(\mathrm{M}=$ metal). In comparing the spectra, we focused our attention on the vibrations of the groups of $\mathrm{C}=\mathrm{O}, \mathrm{COO}^{-}$and $\mathrm{COH}$ when they are in the region 1000 to $2000 \mathrm{~cm}^{-1}$, which is less susceptible to interference from contaminants.

Further, the most stable complex was estimated based on total energy calculations. For lithium citrates, the CA:Li proportion 1:1 should be the most stable. For barium citrates, the estimate indicates that the CA:Ba ratio 3:1 is probably the most stable complex, which is supported by experimental results.

\section{Acknowledgements}

This research was supported by resources supplied by the Center for Scientific Computing (NCC/GridUNESP) of São Paulo State University (UNESP). The authors acknowledge the scholarships UNESP (2010/15588) and Fundação de Amparo à Pesquisa do Estado de São Paulo (FAPESP) (grant 2011/022455) (RMF), Coordenação de Aperfeiçoamento do Pessoal do Ensino Superior (CAPES) (ABN) and Instituto Nacional de Ciência e Tecnologia de Materiais em Nanotecnologia (INCTMN) (CFOG/ABN), and FAPESP for the grant 2007/080720 (PNLF/MM). 


\section{References}

1. Motta M, Deimling CV, Saeki MJ and Lisboa PN Fo. Chelating agent effects in the synthesis of mesoscopic-size superconducting particles. Journal of Sol-Gel Science and Technology. 2008; 46(2):201-207. http://dx.doi.org/10.1007/ s10971-007-1673-0

2. Jafar MH. $\mathrm{LiMn}_{0.3} \mathrm{Co}_{0.3} \mathrm{Ni}_{0.3} \mathrm{Cr}_{0.1} \mathrm{O}_{2}$ cathode materials prepared via sol-gel and combustion methods: a comparison. AIP Conference Proceedings. 2011; 1400(1):280-285. http://dx.doi. org/10.1063/1.3663128

3. Wang DQ, Cao LY, Huang JF and Wu JP. Synthesis and electrochemical properties of $\mathrm{LiV}_{3} \mathrm{O}_{8}$ via an improved sol-gel process. Ceramics International. 2012; 38(4):2647-2652. http://dx.doi.org/10.1016/j.ceramint.2011.11.030

4. Chiang C, Shei CY, Wu SF and Huang YT. Preparation of high-purity T1-based ' 1223 ' superconductor phase by modified Pechini process in water solution. Applied Physics Letters. 1995; 58(21):2435-2437. http://dx.doi. org/10.1063/1.104865

5. Kakihana M. Sol-gel preparation of high temperature superconducting oxides. Journal of Sol-Gel Science and Technology. 1996; 6(1):7-55. http://dx.doi.org/10.1007/ BF00402588

6. Pathak LC and Mishra SK. A review on the synthesis of Y-Ba-Cu-oxide powder. Superconductor Science and Technology. 2005; 18:67-89. http://dx.doi.org/10.1088/09532048/18/9/R01

7. Coey JMD, Viret M and von Molnár S. Mixed-valence manganites. Advances in Physics. 1999; 48(2):167-293. http:// dx.doi.org/10.1080/000187399243455

8. Pullar RO. Hexagonal ferrites: a review of the synthesis, properties and applications of hexaferrite ceramics. Progress in Material Sciences. 2012; 57(7):1191-1334. http://dx.doi. org/10.1016/j.pmatsci.2012.04.001

9. Eerenstein V, Mathur ND and Scott JF. Multiferroic and magnetoelectric materials. Nature. 2006; 442(7104):759-765. PMid:16915279. http://dx.doi.org/10.1038/nature05023

10. Zampieri M, Lazaro SR, Paskocimas CA, Ferreira AG, Longo E and Varela JA. Structural analysis of $\mathrm{Ti}$ and $\mathrm{Pb}$ citrate using NMR and FT-Raman signals and quantum mechanics simulations. Journal of Sol-Gel Science and Technology. 2006; 37:9-17. http://dx.doi.org/10.1007/s10971005-4892-2

11. Coskuner O and Jarvis EAA. Coordination studies of AlEDTA in aqueous solution. Journal of Physical Chememistry A. 2008; 112(12):2628-2633. PMid:18293948. http://dx.doi. org/10.1021/jp7098575

12. Kovács A, Nemcsok DS and Kocsis T. Bonding interactions in EDTA complexes. Journal of Molecular Structure: THEOCHEM. 2010; 950(1-3):93-97. http://dx.doi. org/10.1016/j.theochem.2010.03.036

13. Yang W and Mortier WJ. The use of global and local molecularparameters for the analysis of the gas-phase basicity of amines. Journal of American Chemical Society. 1986; 108(19):57085711. PMid:22175316. http://dx.doi.org/10.1021/ja00279a008

14. Mineva T. Selectivity study from the density functional local reactivity indices. Journal of Molecular Structure: THEOCHEM. 2006; 762(1):79-86. http://dx.doi.org/10.1016/j. theochem.2005.08.044

15. Cruz J, Martínez-Aguilera LMR, Salcedo R and Castro M. Reactivity properties of derivatives of 2-imidazoline: An ab initio DFT study. International Journal of Quantum
Chemistry. 2001; 85(4-5):546-556. http://dx.doi.org/10.1002/ qua. 10018

16. Bronze-Uhle ES, Batagin A No, Lavarda FC and Graeff CFO. Ionizing radiation induced degradation of poly (2-methoxy5-(2-ethyl-hexyloxy) -1,4-phenylene vinylene) in solution. Journal of Applied Physics. 2011; 110:073510. http://dx.doi. org/10.1063/1.3644946

17. Stewart JJP. Optimization of parameters for semiempirical methods V: Modification of NDDO approximations and application to 70 elements. Journal of Molecular Modeling. 1989; 13(12):1173-1213. PMid:17828561 PMCid:PMC2039871. http://dx.doi.org/10.1007/s00894007-0233-4

18. Stewart JJP. MOPAC2009. Colorado Springs: Stewart Computational Chemistry; 2008. Available from: <http:// openmopac.net/>. Access in: 03/02/2011.

19. Parr RG and Yang W. Density-functional theory of atoms and molecules. New York: Oxford University Press; 1989.

20. Dunbar RC, Oomens J, Orlova G and Bohme DK. IRMPD spectroscopic investigation of gas-phase complexes of deprotonated penicillin $\mathrm{G}$ with $\mathrm{Ba}^{2+}, \mathrm{Zn}^{2+}$ and $\mathrm{Cd}^{2+}$. International Journal of Mass Spectrometry. 2011; 308:330-337. http:// dx.doi.org/10.1016/j.ijms.2011.08.018

21. Stephens PJ, Devlin FJ, Chablowski CF and Frisch MJ. $\mathrm{Ab}$ initio calculation of vibrational absorption and circular dichroism spectra using density functional force fields. Journal of Physical Chemistry. 1994; 98:11623-11627. http://dx.doi. org/10.1021/j100096a001

22. Sousa SF, Fernandes PA and Ramos MJ. General performance of density functionals. Journal of Physical Chemistry A. 2007; 111:10439-10452. PMid:17718548. http://dx.doi. org/10.1021/jp0734474

23. Hehre WJ. A guide to molecular mechanics and quantum chemical calculations. California: Wavefunction; 2003.

24. Hay PJ and Wadt WR. Ab initio effective core potentials for molecular calculations. Potentials for $\mathrm{K}$ to $\mathrm{Au}$ including the outermost core orbitals. Journal of Chemical Physics. 1985; 82:299-300. http://dx.doi.org/10.1063/1.448975

25. Feller D. The role of databases in support of computational chemistry calculations. Journal of Computational Chemistry. 1996; 17:1571-1586. http://dx.doi.org/10.1002/ (SICI)1096-987X(199610)17:13\%3C1571::AIDJCC9\%3E3.0.CO;2-P

26. Levine IN. Quantum chemistry. New York: Prentice Hall; 1999.

27. Lewars EG. Computational chemistry: introduction to the theory and applications of molecular and quantum mechanics. New York: Springer; 2010.

28. National Institute of Standards and Technology (NIST). Computational chemistry comparison and benchmark database. Available from: <http://cccbdb.nist.gov/vibscalejust. asp>. Access in: 15/06/2011.

29. Schmidt MW, Baldridge KK, Boatz JA, Elbert ST, Gordon MS, Jensen JH et al. General atomic and molecular eletronic-structure system. Journal of Computational Chemistry. 1993; 14(11):1347-1363. http://dx.doi.org/10.1002/ jcc.540141112

30. Allouche AR. Gabedit: a graphical user interface for computational chemistry softwares. Journal of Computational Chemistry. 2011; 32(1):174-182. PMid:20607691. http:// dx.doi.org/10.1002/jcc. 21600

31. Bode BM and Gordon MS. MacMolPlt: a graphical user interface for GAMESS. Journal of Molecular Graphics and 
Modelling. 1998; 16(3):133-138. http://dx.doi.org/10.1016/ S1093-3263(99)00002-9

32. Kakihana M, Arima M, Nakamura Y, Yashima M and Yoshimura M. Spectroscopic characterization of precursors used in the pechini-type polymerizable complex processing of barium titanate. Chemistry of Materials. 1999; 11(2):438-450. http://dx.doi.org/10.1021/cm9806681

33. Sambrano JR, Zampieri M, Ferreira AG and Longo E. Ab initio study and NMR analysis of the complexion of citric acid with ion lithium. Journal of Molecular Structure. 1999; 493:309318. http://dx.doi.org/10.1016/S0166-1280(99)00268-7

34. Socrates G. Infrared and raman characteristic group frequency. New York: Wiley; 2011.

35. Smith AL. The coblentz society desk book of infrared spectra. Kirkwood: The Coblentz Society; 1982. p. 1-24.

36. Chen HJ, Jian PC, Chen JH, Wang L and Chiu WY. Nanosizedhybrid colloids of poly(acrylic acid)/titania prepared via in situ sol-gel reaction. Ceramics International. 2007; 33(4):643-653. http://dx.doi.org/10.1016/j.ceramint.2005.12.012

37. Kakihana M, Nagumo M, Okanoto M and Kakihana H. Coordination structures for uranyl carboxylate complexes in aqueous-solution studied by IR and carbon-13 NMR spectra. Journal of Physical Chemistry. 1987; 91(24):6128-6136. http:// dx.doi.org/10.1021/j100308a015

38. Stokenhuber M, Mayer H and Lercher JA. Preparation of barium titanate from oxalates. Journal of American Ceramics Society. 1993; 76(5):1185-1190. http://dx.doi. org/10.1111/j.1151-2916.1993.tb03738.x

39. Kakihana M and Yoshimura M. Synthesis and characteristics of complex multicomponent oxides prepared by polymer complex method. Bulletin of the Chemical Society of Japan. 1999; 72(7):1427-1443. http://dx.doi.org/10.1246/ bcsj. 72.1427 